\title{
$\mathrm{J}$

\section{High-Resolution Zero-Field NMR J-Spectroscopy of Aromatic Compounds}

\author{
John W. Blanchard, ${ }^{* \dagger, \ddagger}$ Micah P. Ledbetter, ${ }^{\|}$Thomas Theis, ${ }^{\dagger \neq, \perp}$ Mark C. Butler, ${ }^{\dagger, \ddagger}, \#$ Dmitry Budker, ${ }^{\S, \|}$ \\ and Alexander Pines $*,+$, \\ ${ }^{\dagger}$ Department of Chemistry, University of California at Berkeley, Berkeley, California 94720-3220, United States \\ ${ }^{\ddagger}$ Materials Science Division and ${ }^{\S}$ Nuclear Science Division, Lawrence Berkeley National Laboratory, Berkeley California 94720 , \\ United States \\ "Department of Physics, University of California at Berkeley, Berkeley, California 94720-7300, United States
}

\section{Supporting Information}

ABSTRACT: We report the acquisition and interpretation of nuclear magnetic resonance (NMR) J-spectra at zero magnetic field for a series of benzene derivatives, demonstrating the analytical capabilities of zero-field NMR. The zeroth-order spectral patterns do not overlap, which allows for straightforward determination of the spin interactions of substituent functional groups. Higher-order effects cause additional line splittings, revealing additional molecular information. We demonstrate resonance linewidths as narrow as $11 \mathrm{mHz}$, permitting resolution of minute frequency differences and precise determination of long-range $J$-couplings. The measurement of $J$-couplings with the high precision offered by zero-field NMR may allow further refinements in the determination of molecular structure and conformation.

\section{INTRODUCTION}

Nuclear magnetic resonance (NMR) $)^{1,2}$ is among the most powerful analytical tools available to the chemical and biological sciences for chemical detection, characterization, and structure elucidation. However, the high magnetic fields required for conventional NMR necessitate large, immobile, and expensive superconducting magnets, limiting the use of the technique. As an alternative, detection of NMR at low and zero magnetic fields has attracted attention in recent years as a tool for chemical analysis not limited by the disadvantages of superconducting magnets. Moreover, utilization of nonthermal polarization and encoding techniques which do not rely on chemical shifts has enabled NMR experiments to be performed in the absence of applied magnetic fields. ${ }^{3,4}$

Inductive coils have been used for NMR detection at low fields $(\sim 0.5 \mathrm{G}),{ }^{5,6}$ however, such techniques do not extend fully to zero field and are potentially limited by dramatically increased spectral complexity in the low, but far from zero, field regime, $10^{-7} \mathrm{~T}<B<10^{-3} \mathrm{~T} .^{7,8}$ In the past, acquisition of truly zero-field spectra was time-consuming, requiring cycling between zero and high magnetic field to measure the response point-by-point, and was generally useful only for the simplification of complex spectra of polycrystalline or amorphous solids. ${ }^{9-16}$ More recently, superconducting quantum interference devices (SQUIDs) ${ }^{17}$ have been used to detect $\mathrm{NMR}^{18-20}$ and $\mathrm{MRI}^{21}$ signals at low fields, though SQUID detectors still require cryogenic temperatures. The advent of atomic magnetometers that are sensitive at low or zero

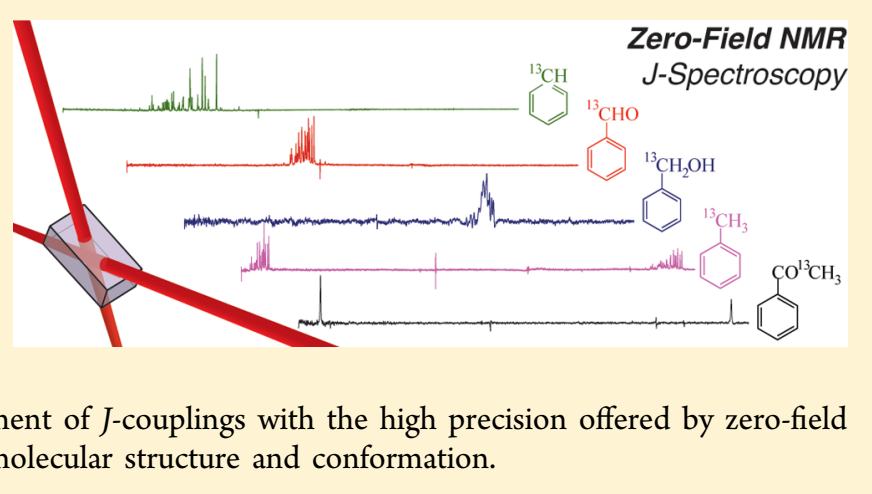

magnetic fields, ${ }^{2-25}$ do not require cryogens, and can be microfabricated, ${ }^{26-28}$ has led to recent experimental demonstrations of zero-field ${ }^{29-32}$ and near-zero-field ${ }^{33}$ NMR and MRI. ${ }^{34-36}$ We achieve high signal-to-noise ratio in zero-field NMR J-spectra using magnetometers based on microfabricated millimeter-scale vapor cells, ${ }^{26}$ which permits the use of 50-250 $\mu \mathrm{L}$ sample volumes, much smaller than typical samples for Earth's-field experiments. Additionally, the use of $\mu$-metal magnetic shielding yields extremely homogeneous fields, allowing for the measurement of linewidths as narrow as 11 $\mathrm{mHz}$, necessary for precise determination of $J$-coupling frequencies.

In the absence of magnetic fields, the Zeeman interaction, and thus the chemical shift, vanishes, leaving an isotropic fluid system to evolve only under electron-mediated scalar couplings ( $J$-couplings) between spins in a molecule. Even in the absence of chemical shifts, the high sensitivity of J-couplings to subtle changes in geometry and electronic structure makes them a valuable source of chemical information. Owing to continued progress in quantum chemical calculations of spin-spin couplings, ${ }^{37-39}$ there now exists a number of techniques for analysis and interpretation of $J$-coupling constants, ranging from empirical Karplus ${ }^{40}$-type equations to newer techniques involving spin-spin coupling density surfaces, ${ }^{41,42}$ double finite perturbation theory calculations, ${ }^{43}$ and decomposition of $J$ -

Received: December 14, 2012

Published: February 7, 2013 


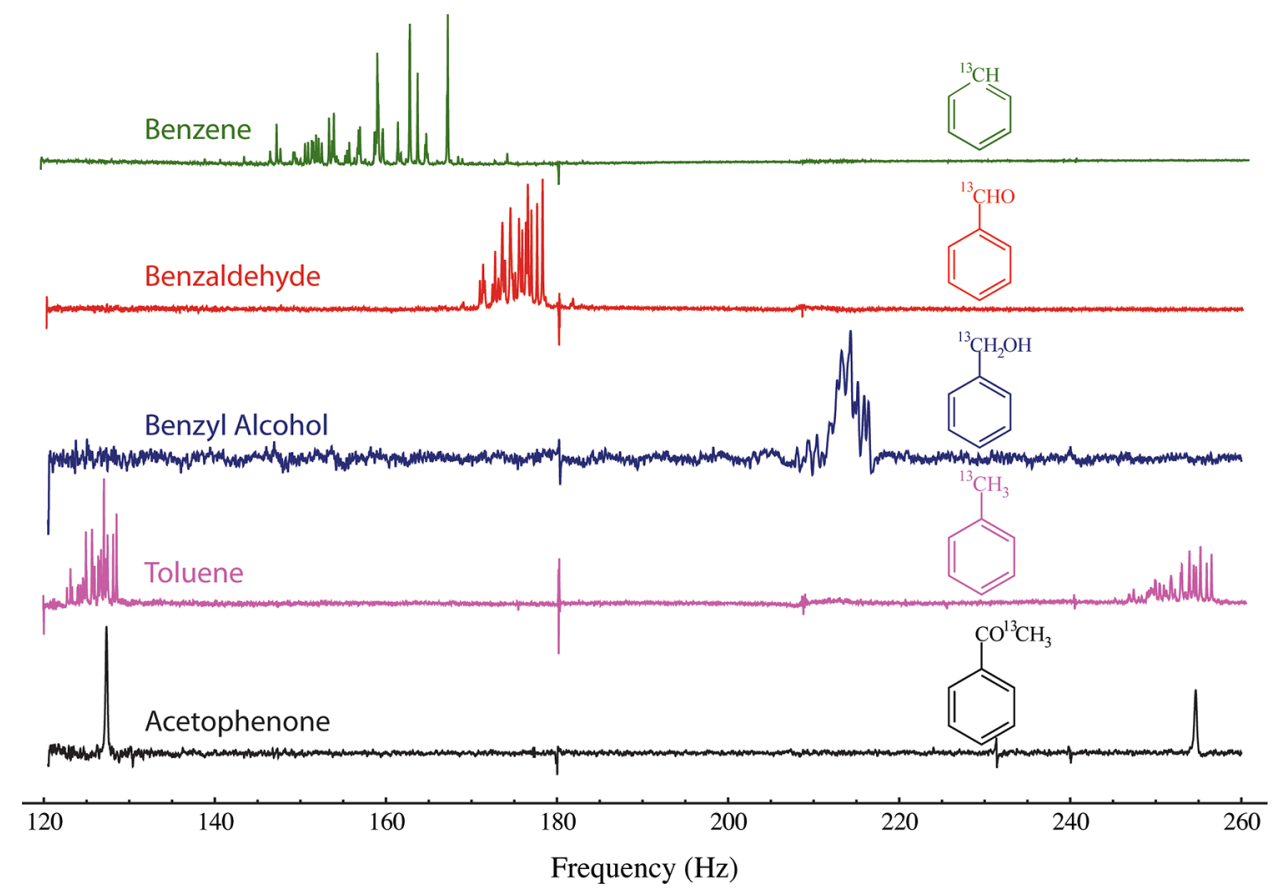

Figure 1. $J$-spectra of a series of benzene derivatives, highlighting the effects of different ${ }^{13} \mathrm{CH}_{n}$ groups and their increasing displacement from the aromatic ring. Clusters of peaks appear at $J$ for $\mathrm{CH}$ groups, at $3 J / 2$ for $\mathrm{CH}_{2}$ groups, and at $J$ and $2 J$ for $\mathrm{CH}_{3}$ groups. The spread of the peaks within the clusters decreases as the distance of the ${ }^{13} \mathrm{C}$ label from the aromatic ring increases. Signals at multiples of $60 \mathrm{~Hz}$ are the result of line noise.

couplings and the Ramsey ${ }^{44}$ terms (Fermi contact, spin dipole, diamagnetic spin orbit, and paramagnetic spin orbit) into orbital contributions. ${ }^{45,46}$ Using these techniques, it has been possible to solve problems of molecular configuration, ${ }^{47-50}$ bond character, ${ }^{51}$ molecular motion, ${ }^{52,53}$ and intermolecular interactions. $^{54,55}$ We have developed a method for the interpretation of zero-field $J$-spectra wherein a preferred basis set for the spins is determined by the one-bond coupling between two heteronuclei, and smaller long-range couplings are treated as perturbations on this primary zeroth-order interaction. For systems consisting of three sets of magnetically-equivalent spins, this analysis leads to simple analytical expressions that fully describe the zero-field spectra.

In order to demonstrate the viability of J-spectroscopy for chemical fingerprinting, we measured high-resolution zero-field $J$-spectra for a series of labeled aromatic molecules: benzene- $-{ }^{13} \mathrm{C}_{1}$, benzaldehyde- $\alpha-{ }^{13} \mathrm{C}_{1}$, benzyl alcohol $-\alpha-{ }^{13} \mathrm{C}_{1}$, toluene- $\alpha-{ }^{13} \mathrm{C}_{1}$, and acetophenone- $\beta-{ }^{13} \mathrm{C}_{1}$. The general qualitative structures of the spectra are consistent with patterns reported previously for simpler spin systems, and furthermore, the spectra for different molecules are unique and easily distinguished from one another. The narrow linewidths (as low as $11 \mathrm{mHz}$ for benzene- ${ }^{13} \mathrm{C}_{1}$ ) permit precise measurement of long-range J-couplings, which encompass a wealth of chemically-relevant information.

\section{RESULTS AND DISCUSSION}

Zero-Field J-Spectra. The zero-field J-spectra for the indicated compounds are shown in Figure 1. For ease of comparison, the vertical axis has been normalized. The signalto-noise ratio in the benzyl alcohol spectrum is somewhat lower on account of broader lines, which we suspect is related to hydrogen bonding. The spectrum for each molecule is dominated by a strong one-bond heteronuclear J-coupling, which is then perturbed by long-range couplings involving the phenyl-ring protons. Differences in molecular structure (i.e., functional groups) give rise to significantly different spectra, with little overlap between spectra for different molecules. The general qualitative structure, being dependent on the strong one-bond coupling, provides information about the substituent functional group (peaks around ${ }^{1} J_{\mathrm{CH}}$ for $\mathrm{CH}, 3 / 2 \times{ }^{1} J_{\mathrm{CH}}$ for $\mathrm{CH}_{2}$, and two sets of peaks around ${ }^{1} J_{\mathrm{CH}}$ and $2 \times{ }^{1} J_{\mathrm{CH}}$ for $\mathrm{CH}_{3}$ ), and the measurement of splittings from long-range J-couplings provides quantitative information about electronic structure and molecular configuration, as discussed below. Because nearly all peaks are resolved (with the exception of the peaks in acetophenone, where the couplings to the methyl group are small), the high-frequency portion of the spectrum (generally $100-300 \mathrm{~Hz}$ ) is sufficient to fully determine all coupling frequencies without the need for consideration of the lower frequency peaks, which are often affected by low-frequency noise and potentially by spectral overlap in mixtures.

Zeroth-Order Energy Levels. The basic qualitative structures of these spectra are consistent with previous work, with peaks at nonzero frequency arising due to J-couplings in molecules containing spins with at least two different gyromagnetic ratios. The simplest examples include ${ }^{13} \mathrm{C}-{ }^{1} \mathrm{H}$ in formate $\left({ }^{13} \mathrm{CHOO}^{-}\right),{ }^{13} \mathrm{C}-{ }^{1} \mathrm{H}_{2}$ in formaldehyde $\left({ }^{13} \mathrm{CH}_{2} \mathrm{O}\right)$, and ${ }^{13} \mathrm{C}-{ }^{1} \mathrm{H}_{3}$ in methanol $\left({ }^{13} \mathrm{CH}_{3} \mathrm{OH}\right)$. We refer to these systems as $\mathrm{XA}, \mathrm{XA}_{2}$, and $\mathrm{XA}_{3}$, respectively, where $\mathrm{X}$ is ${ }^{13} \mathrm{C}$ and $\mathrm{A}_{n}$ represents a set of equivalent protons. Neglecting all couplings other than ${ }^{1} J_{\mathrm{CH}}$, the molecules in this study have spectra that correspond to these simple $\mathrm{XA}_{n}$ systems. The energy levels are given by

$$
E_{0}=\frac{{ }^{1} J_{\mathrm{CH}}}{2}\left[F_{\mathrm{A}}\left(F_{\mathrm{A}}+1\right)-K(K+1)-S(S+1)\right]
$$

where $S=1 / 2$ is the spin quantum number associated with the operator $S$, representing the ${ }^{13} \mathrm{C}$ spin, $K=1 / 3,1,3 / 2, \ldots$ are the possible spin quantum numbers of the operator $\mathbf{K}=\sum_{j} \mathbf{I}_{j}$, 


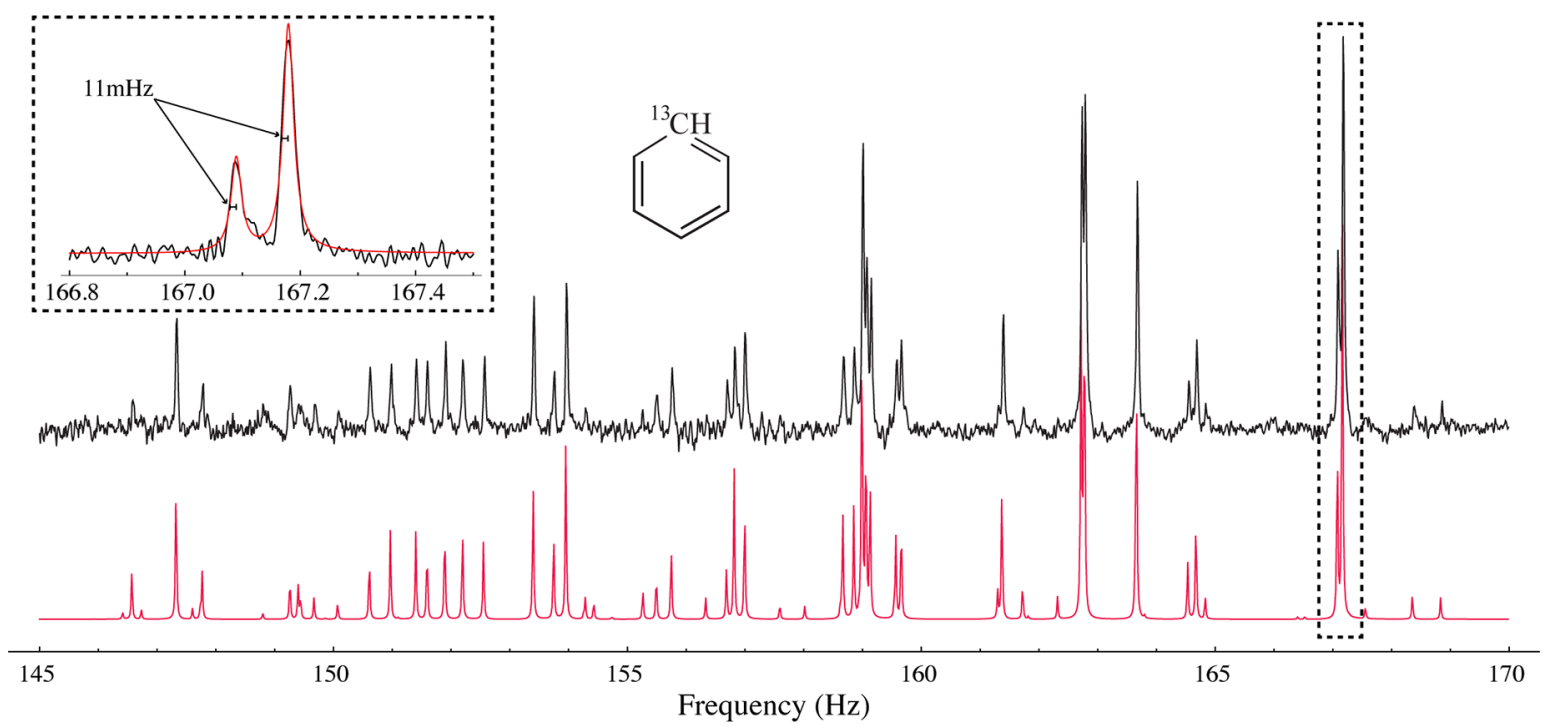

Figure 2. Experimental (upper trace) and simulated (lower trace) spectrum of benzene $-{ }^{13} \mathrm{C}_{1}$ in the neighborhood of ${ }^{1} J_{\mathrm{CH}}$. Inset shows fitting of two high-frequency peaks with $11 \mathrm{mHz}$ half-width at half-maximum, consistent with Fourier resolution limited by 80s acquisition time.

representing the sum of the equivalent proton spins, and $F_{\mathrm{A}}=$ $0,1 / 2,1,3 / 2, \ldots$ are the possible quantum numbers of the total angular momentum $\mathbf{F}_{\mathrm{A}}=\mathbf{K}+\mathbf{S}$.

Because the observable in our experiment is a vector operator, the selection rule for observable coherences is $\Delta F_{\mathrm{A}}$ $=0, \pm 1$. Also, because the zero-field Hamiltonian commutes with both $\mathrm{K}^{2}$ and $\mathrm{S}^{2}, \Delta K=\Delta S=0$. As a result, the allowed nonzero transition frequencies are ${ }^{1} J_{\mathrm{CH}}$ for XA systems, $3 / 2 \times$ ${ }^{1} J_{\mathrm{CH}}$ for $\mathrm{XA}_{2}$ systems, and both ${ }^{1} J_{\mathrm{CH}}$ and $2 \times{ }^{1} J_{\mathrm{CH}}$ for $\mathrm{XA}_{3}$ systems. Similarly, the structure of the zero-field $J$-spectra for benzene- ${ }^{13} \mathrm{C}_{1}$ and benzaldehyde- $\alpha-{ }^{13} \mathrm{C}_{1}$, both of which are zeroth-order XA systems, consists of a cluster of peaks near ${ }^{1} J_{\mathrm{CH}}$. The spectrum for benzyl alcohol- $\alpha-{ }^{13} \mathrm{C}_{1}$, to zeroth order an $\mathrm{XA}_{2}$ system, consists of peaks near $3 / 2 \times{ }^{1} J_{\mathrm{CH}}$, and the spectra of toluene- $\alpha-{ }^{13} \mathrm{C}_{1}$ and acetophenone- $\beta-{ }^{13} \mathrm{C}_{1}$, which are zeroth-order $\mathrm{XA}_{3}$ systems, consist of peaks near ${ }^{1} J_{\mathrm{CH}}$ and $2 \times$ ${ }^{1} \mathrm{~J}_{\mathrm{CH}}$.

Phenyl Perturbations. Beyond the zero-order structure of the zero-field spectra, additional splittings arise due to couplings between the $\mathrm{XA}_{n}$ spin systems and the ring protons. Because all couplings involving the ring protons are at least 15 times smaller than ${ }^{1} J_{\mathrm{CH}}$ (see Supporting Information, SI), these couplings may be treated as a perturbation to the $\mathrm{XA}_{n}$ system. In keeping with notation described previously, the molecules studied in this report may be considered $\left(\mathrm{XA}_{n}\right) \mathrm{BB}^{\prime} \mathrm{CC}^{\prime} \mathrm{D}$ spin systems, where $\mathrm{B}$ and $\mathrm{B}^{\prime}$ are the ring protons in the ortho positions, $\mathrm{C}$ and $\mathrm{C}^{\prime}$ are the protons in the meta positions, and $\mathrm{D}$ is the proton in the para position. It is apparent from Figure 1 that as the ${ }^{13} \mathrm{C}$ label is further displaced from the aromatic ring, the spreading of the peaks decreases, with the greatest peak spreading for benzene $-{ }^{13} \mathrm{C}_{1}$, in which the label is part of the ring, and almost no spreading for acetophenone- $\beta-{ }^{13} \mathrm{C}_{1}$, in which the label is four bonds from the nearest spin on the ring.

Comparison with Simulation. The experimental spectra are in excellent agreement with simulations (performed via numerical diagonalization of the density matrix), as demonstrated in Figure 2 for the high-frequency portion of the zerofield $J$-spectrum of benzene- ${ }^{13} \mathrm{C}_{1}$. The simulated spectrum fully reproduces the multiplet structure, with peak frequencies in agreement to within $\sim 10 \mathrm{mHz}$. Achieving this level of agreement between experiment and simulation required the use of precise values of the benzene J-coupling constants presented in ref 56, which include the influence of ${ }^{13} \mathrm{C}$ isotope effects. Small frequency differences are likely artifacts of limited precision in the literature values for the coupling constants or slight differences in sample preparation. Peak intensities are well-reproduced, with small variations likely arising due to insufficient prepolarization time or nonadiabatic shuttling between the prepolarization field and the detection region.

High Resolution from Narrow Resonances. Because we operate in zero magnetic field, the absolute field homogeneity is exceptional, and extensive magnetic-field shimming is not required. Inhomogeneous broadening is negligible, and spectral lines are extremely narrow, which allows for very precise measurements of line positions and coupling parameters. The zoomed inset in Figure 2 shows the two peaks at 167.089 and 167.179 Hz fit to the sum of two Lorentzians, each with a halfwidth at half-maximum of $11 \mathrm{mHz}$. This line width is comparable to the Fourier resolution of the spectrum, which is limited by the $80 \mathrm{~s}$ acquisition time. It is likely that the intrinsic line width is actually narrower, as the near-zero-field $\mathrm{T}_{2} *$ of unlabeled benzene has been measured on the same instrument to be $21 \mathrm{s.}^{57}$ Since the zero-field $\mathrm{T}_{2}{ }^{*}$ is probably similar to this value, we expect that the intrinsic line width is less than $10 \mathrm{mHz}$.

Consistent Splittings from Phenyl Perturbation. For the $-{ }^{13} \mathrm{C}(=\mathrm{O}) \mathrm{H}$ functional group (XA subsystem) and $-{ }^{13} \mathrm{CH}_{3}$ functional group ( $\mathrm{XA}_{3}$ subsystem), addition of angular momentum yields states with $K=1 / 2$ and $F_{\mathrm{A}}=0,1$. The zeroorder spectrum of these groups includes a peak at frequency ${ }^{1} J_{\mathrm{CH}}$, which corresponds to a transition with $\Delta F_{\mathrm{A}}= \pm 1$ between states with $K=1 / 2$. When the spins of either functional group are weakly coupled to the spins of a phenyl group, the perturbation splits this peak into a consistent multiplet pattern. To demonstrate that the effect of the ring proton perturbation is qualitatively similar for different functional groups, Figure 3 shows a comparison of the $K=$ $1 / 2$ peaks in the spectra of benzaldehyde- $\alpha-{ }^{13} \mathrm{C}_{1}$ (red trace) and toluene- $\alpha-{ }^{13} \mathrm{C}_{1}$ (blue trace), with the benzaldehyde spectrum shifted without scaling to lower frequency for the purpose of 

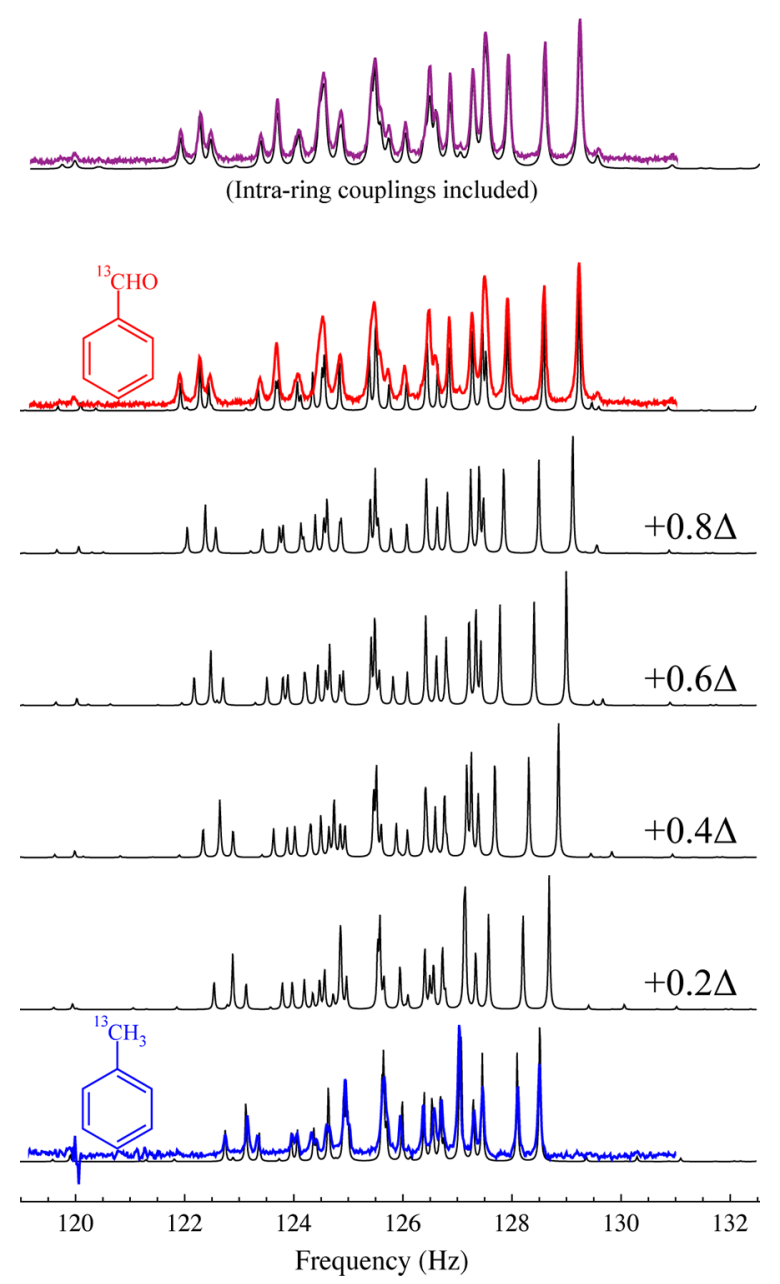

Figure 3. Comparison of $K=1 / 2$ peaks of benzaldehyde- $\alpha-{ }^{13} \mathrm{C}_{1}$ (red trace) and toluene- $\alpha-{ }^{13} \mathrm{C}_{1}$ (blue trace) spectra. The benzaldehyde$\alpha-{ }^{13} \mathrm{C}_{1}$ spectrum has been shifted to lower frequency by $48.86 \mathrm{~Hz}$ for the purpose of illustration, but it has not been scaled. Intermediate simulated spectra are produced by incrementally varying the longrange substituent-to-ring ${ }^{13} \mathrm{C}-{ }^{1} \mathrm{H}$ and ${ }^{1} \mathrm{H}-{ }^{1} \mathrm{H}$ coupling constants by a uniform fraction of the differences between the values for the two molecules $(\Delta)$. The general structure of the peaks is similar because of the identical spin topology, with small quantitative differences arising due to geometric differences and substituent effects. The fit is in reasonable agreement with the benzaldehyde spectrum, even when adjustments to the intraring couplings constants are not included.

illustration. The smooth black traces show simulated spectra, obtained by smoothly interpolating $J$-coupling parameters, as discussed below. While there are small differences in the multiplet structures, many features are present in the spectra of both molecules, for example, the three peaks at the lowerfrequency end of the spectrum (around $123 \mathrm{~Hz}$ for toluene) and the three larger peaks at the higher-frequency end (around $128 \mathrm{~Hz}$ for toluene). The multiplets have a similar structure because the network of couplings involving the ring couplings is $\left(\mathrm{BB}^{\prime} \mathrm{CC}^{\prime} \mathrm{D}\right)$ is topologically identical for any molecule containing a phenyl group. The qualitative similarity of the multiplet patterns facilitates chemical fingerprinting analysis. Variations in the splittings and central frequencies of multiplets allow for differentiation between the spectra of similar molecules.

Physical Significance of Differences Between Benzaldehyde and Toluene. The differences that arise between the
$K=1 / 2$ multiplets in the benzaldehyde- $\alpha-{ }^{13} \mathrm{C}_{1}$ and toluene$\alpha-{ }^{13} \mathrm{C}_{1}$ spectra are associated with important differences between the two molecules. Specifically, the J-coupling constants depend on electronic structure, which in turn depends on substituent electron-donating/withdrawing effects and the spatial orientation of the substituent with respect to the plane of the ring. The variation in the ${ }^{n} J_{\mathrm{CH}}$ couplings is primarily dependent on the electronegativity of the substituent, with a more strongly electron-withdrawing substituent leading to a larger coupling between the substituent ${ }^{13} \mathrm{C}$ and ring protons. The ${ }^{n} J_{\mathrm{CH}}$ couplings in benzaldehyde- $\alpha-{ }^{13} \mathrm{C}_{1}$ are thus larger than in toluene- $\alpha-{ }^{13} \mathrm{C}_{1}$, as the more electronegative aldehyde substituent more strongly attracts the $\pi$-electron density of the aromatic ring, increasing the overlap with the ${ }^{13} \mathrm{C}$ nucleus. The ${ }^{n} J_{\mathrm{HH}}$ benzylic couplings between the substituent protons and the aromatic protons have been studied, ${ }^{58-61}$ and they feature a dependence on the angle by which the substituent protons are rotated out of the plane of the ring. The couplings are given as

$$
\begin{aligned}
& { }^{4} J\left(\mathrm{H}, \mathrm{CH}_{n}\right)=6.90 p\left\langle\sin ^{2} \theta\right\rangle-0.32\left\langle\cos ^{2} \theta\right\rangle \\
& { }^{5} J\left(\mathrm{H}, \mathrm{CH}_{n}\right)={ }^{5} J^{\pi}\left\langle\sin ^{2} \theta\right\rangle+{ }^{5} J\left\langle\sin ^{2}(\theta / 2)\right\rangle
\end{aligned}
$$

and

$$
{ }^{6} J\left(\mathrm{H}, \mathrm{CH}_{n}\right)={ }^{6} J_{90}\left\langle\sin ^{2} \theta\right\rangle
$$

where $\theta$ is the angle by which the substituent $\mathrm{C}-\mathrm{H}$ bond twists out of the benzene plane, $p$ is the mutual atom-atom polarizability, ${ }^{5} J^{\pi}$ is the $\sigma-\pi$ electron contribution to the ${ }^{5} J\left(\mathrm{H}, \mathrm{CH}_{n}\right)$ coupling, ${ }^{5} J^{\sigma}$ is the $\sigma$-electron component, and ${ }^{6} J_{90}$ is the value of ${ }^{6} \mathrm{~J}\left(\mathrm{H}, \mathrm{CH}_{n}\right)$ when the substituent $\mathrm{C}-\mathrm{H}$ bond is perpendicular to the plane of the benzene ring. Key differences between the toluene and benzaldehyde ${ }^{n} J_{\mathrm{HH}}$ couplings arise because of differences in these parameters. The most striking differences are related to the angle $\theta$, and the ${ }^{6} J\left(\mathrm{H}, \mathrm{CH}_{n}\right)$ coupling is an illustrative example. Because the methyl group in toluene rotates freely, the expectation value of $\sin ^{2} \theta$ is 0.5 , for a coupling of $-0.52 \mathrm{~Hz}$, whereas in benzaldehyde, the formyl proton is essentially in the benzene plane, and the coupling is only $-0.018 \mathrm{~Hz}$.

The intermediate calculated spectra in Figure 3 were produced by varying the ${ }^{n} J_{\mathrm{CH}}$ and ${ }^{n} J_{\mathrm{HH}}$ couplings in linear fractional increments between the values for toluene- $\alpha-{ }^{13} \mathrm{C}_{1}$ and benzaldehyde- $\alpha-{ }^{13} \mathrm{C}_{1}$, leaving the intraring couplings constant. The agreement with the benzaldehyde spectrum is good, with the simulation fully reproducing the multiplet structure. Slight disagreements are the result of minute differences in intraring couplings that are affected by differences in aromatic ring electron densities induced by substituent effects. Including these effects, which cause changes of $\sim 0.02-0.2 \mathrm{~Hz}$ (the effects are strongest for the four-bond couplings, followed by the three- and then five-bond couplings), gives full agreement, as indicated by the uppermost traces in Figure 3.

$J$-couplings measured in these zero-field experiments agree with the values determined by high-field NMR (see SI). Because the zero-field spectra generally have enough peaks to determine all $J$-coupling frequencies, and thus provide enough information for chemical identification and elucidation of electronic structure, topology, and spatial configuration, the presence of chemical shifts may not always be necessary for chemical identification and analysis. 


\section{CONCLUSIONS}

Zero-field NMR J-spectroscopy has been demonstrated for a series of benzene derivatives, yielding unique, well-resolved, and information-rich spectra that are well suited for chemical fingerprinting. Long relaxation times and consistent field homogeneity give rise to extremely narrow resonance linewidths and enhanced resolution. The narrow linewidths allow for measurement of $J$-coupling frequencies with greater precision than is typically achieved using other techniques. Conveniently, in the molecules studied here, zero-field spectra are all nonoverlapping, which facilitates analysis of mixtures without the need for higher-dimensional spectroscopy. The existence of consistent qualitative spectral patterns allows for quick assignment of $\left(\mathrm{XA}_{n}\right) \mathrm{BB}^{\prime} \mathrm{CC}^{\prime} \mathrm{D}$ spin systems, so that implementation of search algorithms with zero-field simulations should yield precise J-coupling values. While all samples measured in this study consist of selectively ${ }^{13} \mathrm{C}$-labeled molecules, ideally one would have the capability to measure samples with ${ }^{13} \mathrm{C}$ in natural abundance. This may be achieved by incorporating hyperpolarization techniques or by improvements in magnetometric sensitivity. Regarding the latter, we estimate that the fundamental limits of sensitivity are $\sim 100$ times lower than the present noise level. ${ }^{23}$ Implementation of hyperpolarization techniques should extend the applicability of zero-field NMR experiments to more "normal" (dilute, natural abundance) samples, as has been demonstrated for systems amenable to parahydrogen-induced polarization. ${ }^{3,4}$

Combining this new technique for the precise measurement of electron-mediated scalar couplings with quantum chemistry calculations should provide detailed information about molecular conformation and electronic structure. This contribution supports the development of zero-field NMR as a technique complementary to high-field NMR, enabling precision measurement of couplings. Furthermore, the absence of superconducting magnets in zero-field NMR facilitates cost effectiveness and portability.

\section{MATERIALS AND METHODS}

Sample Preparation. Benzene- ${ }^{13} \mathrm{C}_{1}$, benzaldehyde $-\alpha-{ }^{13} \mathrm{C}_{1}$, benzyl alcohol- $\alpha-{ }_{-13} \mathrm{C}_{1}$, toluene- $\alpha-{ }^{13} \mathrm{C}_{1}$, and acetophenone- $\beta-{ }^{13} \mathrm{C}_{1}$ were obtained from Cambridge Isotopes Laboratory. Samples were degassed via several freeze-thaw cycles under vacuum and flame-sealed in $5 \mathrm{~mm}$ NMR tubes.

Zero-Field NMR Measurements. Samples were thermally polarized in a $2 \mathrm{~T}$ magnet and pneumatically shuttled into a zerofield detection region where the field of the nuclear spins was measured using an alkali-vapor atomic magnetometer. As the sample was shuttled to the detection region, a solenoid produced a guiding field in order to keep the initial magnetization aligned vertically. Once the sample reached the detection region, the solenoid was turned off, and a DC magnetic field pulse with area $\gamma_{\mathrm{H}} B t \approx 4 \pi$ was applied in an orthogonal direction to maximize the $\mathbf{I}_{Z}-\mathbf{S}_{Z}$ components of the density matrix, thus maximizing the signal (see forthcoming work by Butler, et al. for additional details). A more thorough description of the apparatus can be found in refs 29 and 33 .

Spectra for benzene- $-{ }^{13} \mathrm{C}_{1}$, benzaldehyde- $\alpha-{ }^{13} \mathrm{C}_{1}$, toluene- $\alpha-{ }^{13} \mathrm{C}_{1}$, and acetophenone- $\beta-{ }^{13} \mathrm{C}_{1}$ were acquired without heating or cooling the sample, at roughly $35^{\circ} \mathrm{C}$. To compensate for hydrogen-bonding linebroadening effects in benzyl alcohol- $\alpha-{ }^{13} \mathrm{C}_{1}$, the spectrum was taken at $70{ }^{\circ} \mathrm{C}$. The benzene- $-{ }^{13} \mathrm{C}_{1}$ spectrum is the result of averaging 220 transients, each with $100 \mathrm{~s}$ polarization time and $80 \mathrm{~s}$ acquisition time. The benzaldehyde- $\alpha-{ }^{13} \mathrm{C}_{1}$ spectrum was the result of averaging 256 transients, each with $60 \mathrm{~s}$ polarization time and $40 \mathrm{~s}$ acquisition time. The toluene- $\alpha-{ }^{13} C_{1}$ spectrum was the result of averaging 222 transients, each with $60 \mathrm{~s}$ polarization time and $40 \mathrm{~s}$ acquisition time. The acetophenone- $\beta-{ }^{13} \mathrm{C}_{1}$ spectrum was the result of averaging 1180 transients, each with $20 \mathrm{~s}$ polarization time and $16 \mathrm{~s}$ acquisition time. The benzyl alcohol- $\alpha-{ }^{13} \mathrm{C}_{1}$ spectrum was the result of averaging 2300 transients, each with $20 \mathrm{~s}$ polarization time and $8 \mathrm{~s}$ acquisition time. It is worth noting that while these experiments require several hours of signal averaging using thermal polarization at $2 \mathrm{~T}$, improvements in magnetometric sensitivity and sample polarization may significantly reduce acquisition times.

High-Field NMR Measurements. High-field NMR spectra (see SI) of labeled benzaldehyde and toluene were collected on a Bruker Avance $300 \mathrm{MHz}(7.04 \mathrm{~T})$ spectrometer with a Bruker $5 \mathrm{~mm}{ }^{1} \mathrm{H} /{ }^{13} \mathrm{C}$ liquids probe. No ${ }^{13} \mathrm{C}$ or ${ }^{1} \mathrm{H}$ decoupling was performed. Careful FID shimming was performed in order to maximize resolution for the observation of $\mathrm{J}_{\mathrm{CH}} / \mathrm{J}_{\mathrm{HH}}$ splitting patterns. Long acquisitions $(4 \mathrm{~s})$ were performed, and negative Lorentzian line-broadening $(-0.1 \mathrm{~Hz})$ was applied to enhance spectral resolution. The same degassed samples used for the low-field measurements were used for the high-field measurements.

Simulations. Zero-field $J$-spectra were simulated by numerical diagonalization of the density matrix. The time-dependent magnetization was determined by evolving the initial thermally-polarized density matrix under the J-coupling Hamiltonian as described in ref 29. Specific J-coupling frequencies were either taken directly from the literature (as in the case of benzene- ${ }^{13} \mathrm{C}_{1}$ ) or were determined by manually refining literature and/or measured values to optimize agreement with experimental spectra (for benzaldehyde- $\alpha-{ }^{13} \mathrm{C}_{1}$ and toluene- $\alpha-{ }^{13} C_{1}$ ). No iterative fitting algorithms were used, though such methods may allow for easier extraction of high-precision J-coupling frequencies in the future.

\section{ASSOCIATED CONTENT}

\section{S Supporting Information}

Tables of coupling values used for simulated $J$-spectra of benzene- ${ }^{13} \mathrm{C}_{1}$, benzaldehyde- $\alpha-{ }^{13} \mathrm{C}_{1}$, and toluene- $\alpha-{ }^{13} \mathrm{C}_{1}$. Highfield ${ }^{13} \mathrm{C}$ spectra for benzaldehyde- $\alpha-{ }^{13} \mathrm{C}_{1}$ and toluene- $\alpha-{ }^{13} \mathrm{C}_{1}$ without ${ }^{1} \mathrm{H}$ decoupling, which served as a starting point for the determination of ${ }^{n} J_{\mathrm{CH}}$ couplings that were not available in the literature. This material is available free of charge via the Internet at http://pubs.acs.org.

\section{AUTHOR INFORMATION}

\section{Corresponding Author}

pines@berkeley.edu; jwblanchard@berkeley.edu

\section{Present Addresses}

${ }^{\perp}$ Department of Chemistry, Duke University, Durham, NC 27708-0354.

"William R. Wiley Environmental Molecular Sciences Laboratory, Pacific Northwest National Laboratory, Richland, Washington 99352.

\section{Notes}

The authors declare no competing financial interest.

\section{ACKNOWLEDGMENTS}

Research was supported by the U.S. Department of Energy, Office of Basic Energy Sciences, Division of Materials Sciences and Engineering under contract no. DE-AC02-05CH11231 (instrument fabrication and salaries for J.W.B., T.T., M.C.B., A.P.) and by the National Science Foundation under award no. CHE-0957655 (samples and salaries for M.P.L. and D.B.). J.W.B. is also supported by a National Science Foundation Graduate Research Fellowship under grant no. DGE-1106400. We further thank S. Knappe and J. Kitching for supplying the microfabricated vapor cell. 


\section{REFERENCES}

(1) Ernst, R. R.; Bodenhausen, G.; Wokaun, A. Principles of Nuclear Magnetic Resonance in One and Two Dimensions; Oxford University Press: Oxford, U.K., 1987.

(2) Slichter, C. P. Principles of Magnetic Resonance; 3rd ed.; Springer: New York, 1990.

(3) Theis, T.; Ganssle, P.; Kervern, G.; Knappe, S.; Kitching, J.; Ledbetter, M. P.; Budker, D.; Pines, A. Nature Phys. 2011, 7, 571-575.

(4) Theis, T.; Ledbetter, M. P.; Kervern, G.; Blanchard, J. W.; Ganssle, P. J.; Butler, M. C.; Shin, H. D.; Budker, D.; Pines, A. J. Am. Chem. Soc. 2012, 134, 3987-3990.

(5) Appelt, S.; Glöggler, S.; Häsing, F. W.; Sieling, U.; Gordji-Nejad, A. Chem. Phys. Lett. 2010, 485, 217-220.

(6) Blümich, B.; Casanova, F.; Appelt, S. Chem. Phys. Lett. 2009, 477, 231-240.

(7) Appelt, S.; Häsing, F. W.; Sieling, U.; Gordji-Nejad, A.; Glöggler, S.; Blümich, B. Phys. Rev. A 2010, 81, 23420.

(8) Halse, M. E.; Callaghan, P. T.; Feland, B. C.; Wasylishen, R. E. J. Magn. Reson. 2009, 200, 88-94.

(9) Bielecki, A.; Zax, D. B.; Thayer, A. M.; Millar, J. M.; Pines, A. Z. Naturforsch. A 1986, 41, 440-444.

(10) Bielecki, A.; Zax, D. B.; Zilm, K. W.; Pines, A. Rev. Sci. Instrum. 1986, 57, 393-403.

(11) Millar, J. M.; Thayer, A. M.; Bielecki, A.; Zax, D. B.; Pines, A. J. Chem. Phys. 1985, 83, 934-938.

(12) Thayer, A. M.; Millar, J. M.; Pines, A. Chem. Phys. Lett. 1986, $129,55-58$.

(13) Weitekamp, D. P.; Bielecki, A.; Zax, D.; Zilm, K.; Pines, A. Phys. Rev. Lett. 1983, 50, 1807-1810.

(14) Zax, D. B.; Bielecki, A.; Kulzick, M. A.; Muetterties, E. L.; Pines, A. J. Phys. Chem. 1986, 90, 1065-1069.

(15) Zax, D. B.; Bielecki, A.; Zilm, K. W.; Pines, A. Chem. Phys. Lett. 1984, 106, 550-553.

(16) Zax, D. B.; Bielecki, A.; Zilm, K. W.; Pines, A.; Weitekamp, D. P. J. Chem. Phys. 1985, 83, 4877-4905.

(17) Greenberg, Y. S. Rev. Mod. Phys. 1998, 70, 175-222.

(18) Black, B.; Majer, G.; Pines, A. Chem. Phys. Lett. 1993, 201, 550554.

(19) McDermott, R; Trabesinger, A. H.; Muck, M.; Hahn, E. L.; Pines, A.; Clarke, J. Science 2002, 295, 2247-2249.

(20) Trabesinger, A. H.; McDermott, R.; Lee, S. K.; Muck, M.; Clarke, J.; Pines, A. J. Phys. Chem. A 2004, 108, 957-963.

(21) Kelso, N.; Lee, S.-K.; Bouchard, L.-S.; Demas, V.; Mueck, M.; Pines, A.; Clarke, J. J. Magn. Reson. 2009, 200, 285-290.

(22) Allred, J. C.; Lyman, R. N.; Kornack, T. W.; Romalis, M. V. Phys. Rev. Lett. 2002, 89, 130801.

(23) Budker, D.; Romalis, M. Nature Phys. 2007, 3, 227-234.

(24) Dang, H. B.; Maloof, A. C.; Romalis, M. V. Appl. Phys. Lett. 2010, 97, 151110.

(25) Kominis, I. K.; Kornack, T. W.; Allred, J. C.; Romalis, M. V. Nature 2003, 422, 596-599.

(26) Knappe, S.; Gerginov, V.; Schwindt, P. D. D.; Shah, V.; Robinson, H. G.; Hollberg, L.; Kitching, J. Opt. Lett. 2005, 30, 2351-3.

(27) Shah, V.; Knappe, S.; Schwindt, P. D. D.; Kitching, J. Nat. Photonics 2007, 1, 649-652.

(28) Romalis, M. V. Nat. Photonics 2007, 1, 613-614.

(29) Ledbetter, M. P.; Crawford, C. W.; Pines, A.; Wemmer, D. E.; Knappe, S.; Kitching, J.; Budker, D. J. Magn. Reson. 2009, 199, 25-29. (30) Savukov, I. M.; Romalis, M. V. Phys. Rev. Lett. 2005, 94, 123001.

(31) Theis, T.; Ganssle, P.; Kervern, G.; Knappe, S.; Kitching, J.; Ledbetter, M. P.; Budker, D.; Pines, A. Nat. Phys. 2011, 7, 571-575.

(32) Theis, T.; Ledbetter, M. P.; Kervern, G.; Blanchard, J. W.; Ganssle, P. J.; Butler, M. C.; Shin, H. D.; Budker, D.; Pines, A. J. Am. Chem. Soc. 2012, 134, 3987-3990.

(33) Ledbetter, M. P.; Theis, T.; Blanchard, J. W.; Ring, H.; Ganssle, P.; Appelt, S.; Blümich, B.; Pines, A.; Budker, D. Phys. Rev. Lett. 2011, 107, 107601.
(34) Savukov, I. M.; Zotev, V. S.; Volegov, P. L.; Espy, M. A.; Matlashov, A. N.; Gomez, J. J.; Kraus, R. H., Jr. J. Magn. Reson. 2009, 199, 188-191.

(35) Xu, S.; Crawford, C. W.; Rochester, S.; Yashchuk, V.; Budker, D.; Pines, A. Phys. Rev. A 2008, 78, 013404.

(36) Xu, S.; Yashchuk, V. V.; Donaldson, M. H.; Rochester, S. M.; Budker, D.; Pines, A. Proc. Natl. Acad. Sci. U.S.A. 2006, 103, 1266812671.

(37) Vaara, J.; Jokisaari, J.; Wasylishen, R. E.; Bryce, D. L. Prog. Nucl. Magn. Reson. Spectrosc. 2002, 41, 233-304.

(38) Helgaker, T.; Jaszuński, M.; Pecul, M. Prog. Nucl. Magn. Reson. Spectrosc. 2008, 53, 249-268.

(39) Harris, R. K. Nuclear Magnetic Resonance Spectroscopy: A Physicochemical View; Longman Scientific and Technical: England, 1986.

(40) Karplus, M. J. Chem. Phys. 1959, 30, 11.

(41) Soncini, a.; Lazzeretti, P. J. Chem. Phys. 2003, 118, 7165.

(42) Soncini, a.; Lazzeretti, P. J. Chem. Phys. 2003, 119, 1343.

(43) Malkina, O. L.; Malkin, V. G. Angew. Chem., Int. Ed. Engl. 2003, $42,4335-8$

(44) Ramsey, N. F. Phys. Rev. 1953, 91, 303-307.

(45) Cremer, D.; Gräfenstein, J. Phys. Chem. Chem. Phys. 2007, 9, 2791-816.

(46) Wu, A.; Cremer, D. Phys. Chem. Chem. Phys. 2003, 5, 4541.

(47) Bifulco, G.; Dambruoso, P.; Gomez-Paloma, L.; Riccio, R. Chem. Rev. 2007, 107, 3744-79.

(48) Krivdin, L. B.; Khutsishvili, S. S.; Shemyakina, O. A.; Contreras, H.; Mal, A. G.; Trofimov, B. A. Magn. Reson. Chem. 2007, 45, 758765 .

(49) Chernyshev, K. A.; Krivdin, L. B.; Larina, L. I.; Konkova, T. V.; Demina, M. M.; Medvedeva, A. S. Magn. Reson. Chem. 2007, 45, 661666.

(50) Krivdin, L. B. Annu. Rep. NMR Spectrosc. 2007, 61, 133-245.

(51) Sutter, K.; Autschbach, J. J. Am. Chem. Soc. 2012, 134, 1337413385.

(52) Pietrzak, M.; Benedict, C.; Gehring, H.; Daltrozzo, E.; Limbach, H.-H. J. Mol. Struct. 2007, 844-845, 222-231.

(53) de la Lande, A.; Fressigné, C.; Gérard, H.; Maddaluno, J.; Parisel, O. Chem.-Eur. J. 2007, 13, 3459-69.

(54) Grzesiek, S.; Cordier, F.; Jaravine, V.; Barfield, M. Prog. Nucl. Magn. Reson. Spectrosc. 2004, 45, 275-300.

(55) Pecul, M.; Sadlej, J.; Helgaker, T. Chem. Phys. Lett. 2003, 372, 476-484.

(56) Chertkov, V.; Sergeyev, N. M. J. Magn. Reson. 1983, 52, 400406.

(57) Ledbetter, M. P.; Pustelny, S.; Budker, D.; Romalis, M. V.; Blanchard, J. W.; Pines, A. Phys. Rev. Lett. 2012, 108, 1-5.

(58) Schaefer, T.; Sebastian, R.; Penner, G. H. Can. J. Chem. 1985, 63, 2597-2600.

(59) Barfield, M.; Fallick, C. J.; Hata, K.; Sternhell, S.; Westerman, P. W. J. Am. Chem. Soc. 1983, 105, 2178-2186.

(60) Laatikainen, R. Magn. Reson. Chem. 1986, 24, 588-594.

(61) Kolehmainen, E.; Laatikainen, R.; Kral, V. Magn. Reson. Chem. 1986, 24, 498-502. 